\section{STATUS LYMPHATICUS FROM THE CLINICAL STANDPOINT.}

By H. C. CAMERON, M.D., F.R.C.P., PHYSICIAN TO THE DEPARTMENT FOR DISEASES OF CHILDREN,
GUY'S HOSPITAL.

I shall not spend time in describing the condition of status lymphaticus as displayed in the post-mortem room. The appearances are familiar to all; their interpretation alone is in dispute. Two contrary opinions appear to have their adherents. I believe that neithei sufficiently explains the known facts, and desire to suggest a third.

The condition has generally been regarded as a peculiar and somewhat rare inborn anomaly, and its existence has been frequently advanced in the coroners' courts and elsewhere as an explanation for the sudden death of a child or young person which otherwise appeared inexplicable.. The condition, however, at least among the faulty children of our London poor, is no rarity. In children dying suddenly, whether, for example, as the result of street accidents or of some virulent and rapidly fatal infection-in children, that is to say, whose bodies have not been emaciated and dehydrated by prolonged illness-it is found in the post. mortem room of Guy's Hospital in a proportion of more than 40 per cent. It is possible and probably right to argue that in the case of the virulent and fatal infections the undue rapidity of death has been determined by the lowered resistance of these anomalous children, but the high proportion in which death is due to accident permits of no doubt as to the frequency of its occurrence during life.

Other observers, impressed by the frequency of the condition in the post-mortem room, and noting how plump and rounded the bodies of such children appear, have main. tained that the large glands and the prominent lymphoid follicles represent the normal condition of health. It is said at Guy's Hos. pital that this was the teaching of Sir Samuel Wilks. If so, we must be prepared to accept the view that adenoid vegetations, enlarged tonsils, and enlarged cervical glands also come within the category of the normal, for with hardly an ex. ception the overgrowth of the deep-seated lymphatic apparatus is accompanied by the enlargement of these more superficial structures.

I suggest that no matter. where the Iymphatic glandular tissue is hypertrophied the explanation is the same, and that the bypertrophy may be regarded as the evidence, !persisting after death, of chronic irritation in the corresponding mucous membranes by persistent, though perhaps quiescent, catarrhal infection. . After death the mucous membranes themselves may show no trace of catarrhal reaction; the enlarged glands remain as evidence of its presence during life.

- It is not difficult, I think, to recognize during life a very common condition to which wf may give the name of the " status catarrhalis,"* and which is the clinical analogue of the post-mortem condition status lymphaticus. Children who show this condition suffer almost continuously from a succession of catarrhs. Skin, conjunctiva, the mucous membranes of ear, nose, nasopharynx, bronchi, intestine, are alike involved in chronic catarrhal processes, which from time to time show exacerbations, with the result that hardly a week passes without a pyrexial attack. In typical examples of this condition the children are fat and watery, with rounded, exaggeratedly infantile bodies. The feet and hands are cold and blue. The hair is sparse, dry, and irregular in its growth, so that the margin of the hairy

* The condition has been frequently described under different names. Czerny has called it the exudative diathesis, a name which perhaps fathers called it struma or scrophula an etiological factor. Our foretime came to apply only to the compares which in the course of which a secondary infection with tubercle had manifested itself. scalp lacks definition. The skin on the cheelss and on the point of the chin is reddened and infiltrated with papular eczema. There is often intertrigo behind the ear or in the limb flexures. The upper lip is red and sore from the irritation of the chronic nasal discharge. Mouth breathing is the rule, because of the large tonsils and adenoid vegetations, witn large everted open lips, which often show cracks and fissures. The chest, for the same reason, is narrow and contracted, and contrasts with the swollen and often pigmented abdomen. The bowels are apt to be loose and often contain mucis. The conjunctiva is reddened and injected. In boys preputial irritation is common, in girls vaginal catarrh. Otitis media is very frequent. The teeth decay early and show circular caries. Urticaria papulata is often a constant symptom. " Tempers are usually violent, the intelligence below the normal, so that some have been sent to me as cretins, and the appetite very large.

The symptom, however, upon which I lay most stress is the extreme wateriness of the tissues in these children: Although they present, because of their plump aspect, a fictitious appearance of health, which commonly entirely. deceives their parents as to their well-being, they are in reality atrophic children who have retained an undue amount of fluid in the water dépôts of the body. The nutrition of the skin, teeth, hair, and all the mucous membranes, and their resistance to infection, has beex profoundly lowered. If, however, a period of higher pyrexia supervenes, from exacerbation of the catarrh in one or more situations, the water is rapidly turned out of the body, and the true atrophy of the child is un. masked. Chart I shows the weight of a boy upon thirty-one successive days. I admitted him for observation, when in his usual state of health, and on the thirty-first day his father removed him because he said there was nothing the matter with the child, and that I was experimenting on him. He had as a rule a huge appetite, but on admission he was homesick and would not eat. In the first twenty-four hours his weight fell nearly $40 \mathrm{oz}$. as a result of relative starvation, a fall due not, of course, to tissue loss but to loss of water. After one week his appetite returned; he began to eat 8,10 , and 12 ounces of bread in the day, and in addition took one pint of milk. In
the second week he regained the water, and his weight rose $3 \mathrm{lb}$. in seven days. Thereafter it remained on the higher level, with considerable daily fluctuations. The tem. perature curve is placed above, showing its persistent slight irregularity even in health. I published these observations in a clinical lecture in the Guy's Hospital Gazette in December, 1916. Two months.later the boy was admitted to Guv's Hospital moribund, and died apparently on the

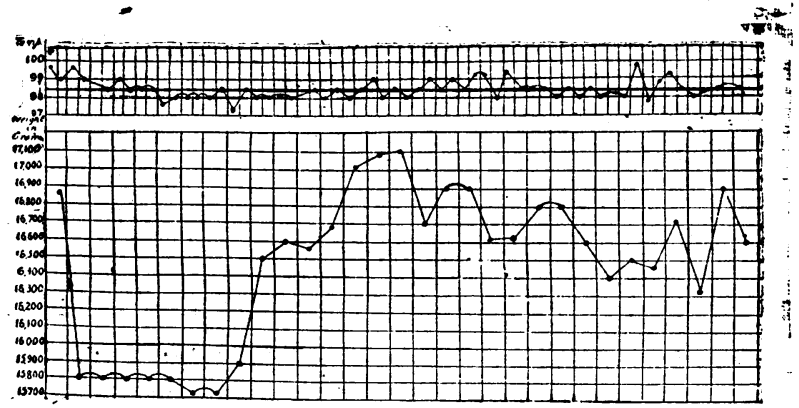

Chart I.

second day of an attack of diphtheria. Post mortem a very extreme degree of status lymphaticus was seen. I had under observation at the same time his younger brother, whose condition was very similar. I do not know what became of this younger brother, but the mortality among such children from diphtheria, measles, and whooping. cough is very large. 
Clinically we meet with examples of the status catarrh. alis both before and after dehydration; in the former case the children are active and lusty enough, in the latter prostrate and complaining. Persistent dehydration is, however, ultimately accompanied by disappearance of the overgrown lymphoid tissue and by a decline in the severity of the catarrhal symptoms. So that, in a sense perhaps, we may regard the higher pyrexial reaction which destroys appetite and produces dehydration as a reaction in the interests of the child. We are familiar with the fact that an acute and spreading eczema is seldom to be seen but in a fat and watery child. If eczema persists in a thin child, it is dry, chronic, and of little intensity. Mothers have observed how rapidly pyrexia clears the skin of eczema. The eczema has struck inwards, they say, as usual ob serving well, but interpreting faultily. Evidence is not wanting that catarrhs of the mucous membranes behave in the same way as the catarrh of the skin. I recall a little boy of six, admitted with a pyrexial illness which the autopsy six weeks later showed to be infective endocarditis. He showed nearly all the signs of what I have called the status catarrhalis. Yet day by day, as his body became emaciated and dehydrated by prolonged pyrexia, one could watch the nasal discharge cease, the succulent mucous membrane become dry, the tonsils and cervical glands steadily diminish, until at death no trace of enlargement could be distinguished.

In the treatment of the status catarrhalis I feel sure that we must direct our efforts towards achieving a less watery habit of body. The condition is dependent, no doubt, upon hereditary factors to some extent; but it develops unchecked only when hygiene is faulty, and when the diet is excessive and unsuitable.

It is the last point that I wish to deal with more precisely. As a result of the economic policy of the last fifty years, which has been directed towards maintaining a cheap loaf and cheap sugar, the children of our poor are fed almost entirely upon bread and sugar. Of these some eat enormously and almost continuously. A mother and six children, for example, habitually consumed $10 \mathrm{lb}$. of bread a day. Children of six years of age will sometimes eat 16 or $18 \mathrm{oz}$. of bread in the day. I have verified this by careful weighing in the ward. I believe that the condition which I describe is very elosely dependent upon this enormous consumption of carbohydrate, so vastly in excess of the requirements of the body.

To illustrate this I reproduce four charts.

Chart II is taken from a normal child aged 15 months, suffering from a slight and transient nasal catarrh. Note (1) that the ing from a slight and transient nasal catarrh. Note (1) that the the temperature adheres closely to the normal line; (3) that the

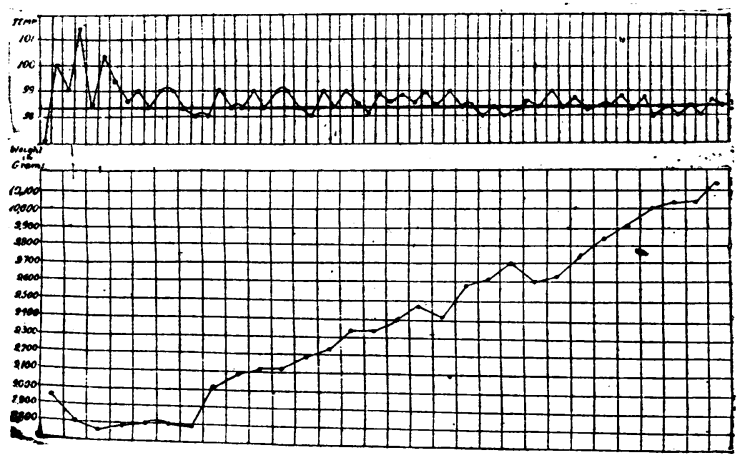

Chart II.

curve of the daily weighing shows a complete absence of large fluctuations.

Chart III shows similar curves, from a child, aged 3, with status catarrhalis, fat and watery, with a huge appetite. The average daily consumption of bread was $15 \mathrm{oz}$. In addition one pint of milk was given.

Note in contrast to Chart I (1) the irregular temperature curve, (2) the fluctuation in the line of the weight curve. On one occasion there was a sudden gain of $24 \mathrm{oz}$. in the day.

Chart IV is a curve from a child, aged 2, so watery that the dorsum of hands and feet pitted on pressure. For the first three weeks a milk diet was given, in the last week large guantities of bread in addition.

(2) the rapid fall in weight from excretion of fluid with the loss of cedema (the fall was equal to one-tenth of the body weight),
(3) the slight genuine gain in weight in the next two weeks (4) the rapid fictitions rise in the last week due to retention of water.

Chart $V$ is a curve from a child, aged 4 , with status catarrh. alis. Till the point marked he was given as much bread he would eat. For the first few days he was homesick and had

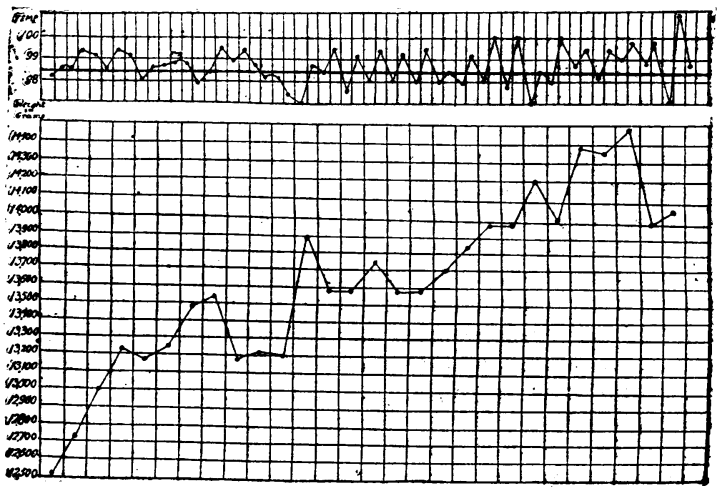

Chart iII.

no appetite: Later he ate very largely. At $A$ he was given a spare diet of milk, fish, eggs, green vegetables, and fruit. Note (1) the unstable temperature, (2) the rapid rise in weight while bread was taken freely, and (3) the initial fall in weight after the change of diet.

In suitable cases I believe that the greatest good can be achieved by persisting for many months in a carefully controlled diet in which the starches and sugars are

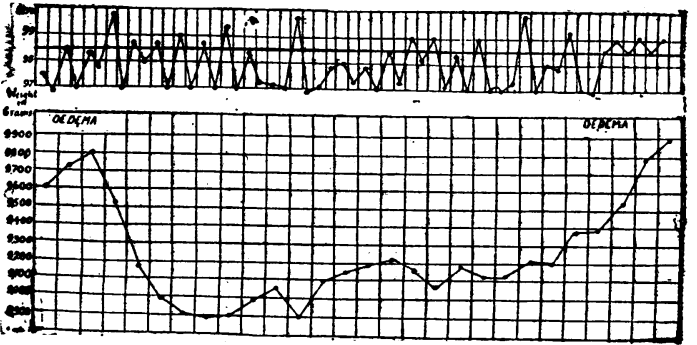

Chart IV.

reduced to a minimum. I have had most success with a diet of skim milk, meat, fish, eggs, green vegetables, and fruit, calculated so as just to cover the physiological needs of the child and controlled by its effect upon the visible catarrhs and palpable glands.

My next point is that if, in this class of case, the vulnerability of the mucous membranes is due to a faul

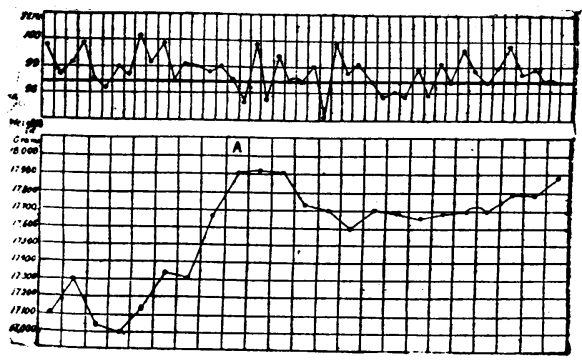

Chart $\nabla$.

of nutrition such as I suggest, it follows that local treatment alone is little likely to be efficacious. I have collected a considerable number of cases in which local measures have been steadily pursued for a long time, sometimes for years, without improvement; which have yielded within a few months to dietetic measures on the lines indicated.

In conclusion, I wish to bring forward for criticism one further suggestion. I believe that the status catarrhalis has a very intimate relation to that somewhat indefinite disorder of later childhood which we call rheumatism; watching many of the children from year to year I find it 
impossible to avoid the conclusion that the rheumatic symptoms are added at the moment when the catarrhal non-pyogenic organisms pass the barrier of the hypertrophied lymphoid tissue and infect the blood stream. Then the child, losing suddenly his watery infantile aspect and taking on a longer and slimmer growth, is said to suffer from growing pains

That the irritated glands are more prone to become infected with tubercle goes almost without saying. The children, as a result of faults of hygiene and of diet, suffer from a lowered resistance to infections of all degrees of severity. Prone to chronic and mild catarrhal processes, they also fall a prey more readily to more severe infections, amongst which rheumatism and tubercle occupy the first place, while measles, diphtheria, and pneumococcal infections often produce a fatal result at the very onset of the disorder, sometimes before the usual post-mortem appearances have had time to develop. Of sudden death from pressure of an enlarged thymus, the so-called mors thymica, I have not spoken. I have no knowledge of the condition, and $I$ am in doubt whether it exists at all.

To sum up I suggest-

Conclustons.

1. That the lymphoid overgrowth so commonly found post mortem in clilidiren is no more than an enlargement from the irritation of chronic catarrh in the corresponding mucous membranes.

2. That such children during life show evidence of faulty nutrition or infection of all epithelial structures, hair skin, teeth, conjunctiva, and the mucous membranes of respiratory and intestinal tracts.

3. That there is usually present a characteristic wateriness of the tissues, which is dependent to some extent upon excessive carbohydrate feeding, and which is the main cause of the vulnerability to infection.

4. That local treatment of the catarrhs alone is likely to be inefficacious, and must be accompanied by a systematic attempt to achieve dehydration and improve the nutrition of the tissues.

5. That the status catarrhalis in the sense defined is a predisposing cause of rheumatism and tubercle, and carries with it a liability to sudden death at the onset of virulent infections, such as pneumococcal infections, measles, or diphtheria.

\section{OBSTRUCTION OF THE URETER BY AN ABNORMAL RENAL VESSEL.} BY

\section{R. P. ROWLANDS; M.S., F.R.C.S.}

Suggeon to Gor's Hospital; Captarn R.A.M.C.(T.).

THE object of this paper is to draw attention to the importance of kinking of the ureter over an abnormal vessel as a cause of hydronephrosis and stone in the kidney. I have met this condition eight times in the last eight years. Congenital stricture at the junction of the ureter and pelvis of the kidney is another important and more frequent cause, which will be more fully considered, with illustrative cases, in another article.

These abnormalities will be found to be not uncommon if the pelvis and ureter are carefully examined as a routine and essential part of every operation on the kidney. Nephropexy should never be performed until obstruction of the ureter as a cause of the symptoms has been definitely excluded. Many failures of nephropexy, like Case vi, are undoubtedly due to mistaken diagnosis. Simila:ly nephrolithotomy is not likely to be permanently successful if the stone has been caused by an obstruction of the ureter which is not discovered and relieved at the operation. In two cases illustrating this paper-Cases III and vir-stones had formed behind the obstraction caused by an abnormal renal vessel. The early recognition and treatment of this condition relieves the patient from frequent and disabling attacks of pain, and the kidney from gradual destruction.

\section{Pathology}

The main renal vessels and their branches pass in front of the renal pelvis to reach the hilus of the kidney, but one of the four or five branches of the renal artery usually passes behind the pelvis, as a rule at too high a level to obstruct the outlet into the ureter.

Occasionally, however, an abnormal renal vessel crosses the origin of the ureter on its way to the lower pole of-the kidney. This is usually an abnormal posterior branch of the renal artery. Sometimes a similar tributary of the renal vein and sometimes a vein and an artery run together. Rarely an additional renal artery runs from the aorta to the lower end of the kidney. As a rule, the offending vessel lies behind, and hitches up the origin of the ureter.

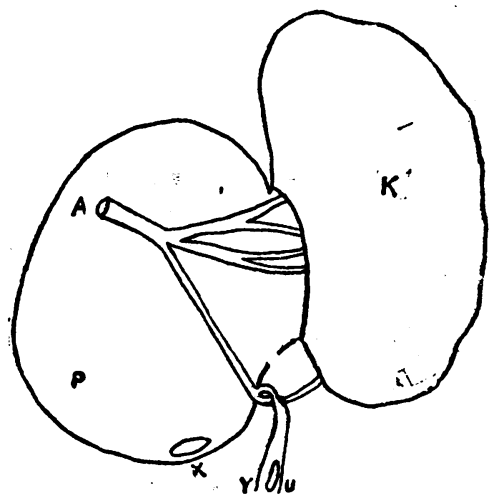

Diagram of obstruction of a ureter by an abnormal vessel. $K$, Anterior view of left kidney; $P$, distended pelvis: $A$, renal artery. a branch of which hitches up the ureter, $\sigma$ at its origin. The division of the abnormal vessel is sufticient in mild cases, but 8 plastic operation or a short circuit at $X$ and $Y$ is necessary in

The abnormality is a little more common on the left side; in five out of my eight cases the left ureter was affected. In some the abnormal vessel crosses the ureter without obstructing it, but when the kidney is unusually movable some obstruction is likely when the trunk is in the vertical position. This leads to gradual dilatation of the pelvis. Any dilatation and pouching of the pelvis is likely to increase the tension upon the artery, and thereíore to increase the obstruction. In the course of time, as the pelvis becomes more and more distended and the heavier kidney descends more, the tense blood ressel exerts more and more pressure on the ureter, inflaming it, and at last causes a real stricture at the site of constriction (Cases IIr, IV, V, VI, and VIII). Many years ago three good specimens were placed in Guy's Hospital Museum-Nos. 1693, 1694, and 1695. Sir Henry Morris figures two of them in his classical work. ${ }^{1}$

These consist of :

\section{Symptoms.}

1. Attacks of renal colic of increasing frequency and severity, which may go on for year's before the condition is recognized. As a rule, the patients are over 25 before an operation is considered. Exercise is apt to bring on these attacks of sudden, characteristic, spasmodic, and severe pain, which run from the loin downwards and inwards into the groin, and often into the testicle, which may be retracted. In some cases the pain runs down the front of the thigh; in a few it does not extend to the groin, but is a semi-girdle pain about the level of the umbilicus. As a rule, the pain is intolerable, the patient cannot sleep or lie still, nausea or romiting are often associated with it, and there may be faintness, pallor, or even shivering. Generally there is some abnormality of micturition, usually increased frequency, irritability, and, perhaps, strangury. At first the urine during and after an attack is normal; later it may be albuminous or even contain blood or pus. Usually there is deep tenderness in the loin, and sometimes a swelling can be felt descending below the costal margin on deep respiration. Early attacks are often far less severe. Sometimes there is shivering and, in late cases, a rise of temperature.

2. In the interval between the attacks there are at times a dull ache, a sense of fullness or dragging pain in the loin.

3. In time the general health deteriorates and the kidney becomes chronically enlarged and tender.

\section{Diagnosiz.}

A. Renal colic has to be distinguished from other severe pains in this region, especially appendicular, biliary, intestinal, and pancreatic colic. Appendicular is 